\title{
NONLINEAR ELLIPTIC PROBLEMS. II
}

\author{
BY FELIX E. BROWDER
}

Communicated October 25, 1963

In a preceding note [1], we proved an existence theorem for solutions of variational boundary value problems for strongly elliptic nonlinear systems of the form

$$
A u=\sum_{|\alpha| \leqq m} D^{\alpha} A_{\alpha}\left(x, u, \cdots, D^{m} u\right)
$$

with $A_{\alpha}$ having at most polynomial growth, by applying a general theorem concerning nonlinear functional equations in reflexive Banach spaces. Our result in [1] extended and generalized results announced earlier by M. I. Višik $[6 ; 7 ; 8]$ and obtained by more concrete analytic arguments. Višik's detailed account of his results which has just appeared in [9] has one feature which goes beyond the framework of methods developed in [1], namely that the hypotheses of strong ellipticity or monotonicity which are assumed involve only the variation of $A_{\alpha}$ with respect to the highest order derivatives and not the lower order derivatives of $u$.

It is our object in the present note to announce some results which constitute an extension of our preceding methods to cover this point. The detailed proof of these results will appear in [4].

1. We use the notation of our preceding note [1].

THEOREM 1. Let $\Omega$ be a bounded smoothly bounded open set in $R^{n}$ with boundary $\Gamma, A$ a system of $r$ differential operators of order $2 m$ acting on $r$-vector functions $u=\left(u_{1}, \cdots, u_{r}\right)$ and having the form

$$
A u=\sum_{|\alpha| \leqq m} D^{\alpha} A_{\alpha}\left(x, u, \cdots, D^{m} u\right) .
$$

Let $\zeta=\left\{\zeta_{\alpha} ;|\alpha| \leqq m\right\}, \eta=\left\{\eta_{\beta} ;|\beta| \leqq m-1\right\}$ be complex vectors. Let $E_{\alpha}(x, \eta, \zeta)$ be functions such that

$$
A_{\alpha}(x, \zeta)=E_{\alpha}(x, \zeta, \zeta)
$$

for all $\zeta$, where $E_{\alpha}$ is measurable in $x$ and continuous in $(\eta, \zeta)$. Suppose that

$$
\left|E_{\alpha}(x, \eta, \zeta)\right| \leqq c\left\{\sum_{|\gamma| \leqq m}\left|\zeta_{\gamma}\right|^{p-1}+\sum_{|\gamma| \leqq m-1}\left|\eta_{\gamma}\right|^{p-1}+1\right\}
$$

for a given exponent $p>1$. 
Let $V$ be a closed subspace of $W^{m, p}(\Omega)$. On $V \times V \times V$, we define the function

$$
e(u ; v, w)=\sum_{|\alpha|_{\S} m}\left\langle E_{\alpha}\left(x,\left\{D^{\gamma} u\right\},\left\{D^{\gamma_{v}}\right\}\right), D^{\alpha_{w}}\right\rangle .
$$

Suppose that:

(a) For each $N>0$ and a fixed $s$ with $s^{-1}>p^{-1}-n^{-1}$, there exists a function $C_{N}(r)$ continuous on $R^{1}, C_{N}(r)>0$ for $r>0, \lim C_{N}(r)=+\infty$ as $r \rightarrow+\infty$, such that

$$
\operatorname{Re}\{e(u ; v, v-w)-e(u ; w, v-w)\} \geqq C_{N}\left(\|v-w\|_{m, p}\right)\|v-w\|_{m, p}
$$
for $\|u\|_{m-1, s} \leqq N$ and all $v, w \in V$.

(b) There exists a function $c(r)$ on $R^{1}$ with $\lim c(r)=+\infty$ as $r \rightarrow \infty$ such that

$$
\operatorname{Re}\{e(u ; u, k u)\} \geqq C\left(\|u\|_{m, p}\right)\|u\|_{m, p}
$$

for all $u \in V, k \geqq 1$.

Then for every $f$ in $V^{*}$, there exists $u$ in $V$ such that

$$
a(u, v)=e(u ; u, v)=(f, v)
$$

for all $v$ in $V$.

Theorem 2. Let $X$ be a reflexive Banach space, $X^{*}$ its conjugate space, $(w, u)$ the pairing between w in $X^{*}$ and $u$ in $X$. Let $Y$ be a second Banach space such that $X$ can be identified with a subset of $Y$ and the injection map is compact linear.

Let $G$ be a mapping (not necessarily linear) of $Y \times X$ into $X^{*}$ and for each $u$ in $Y$, let $G_{u}$ be the mapping of $X$ into $X^{*}$ given by $G_{u}(v)$ $=G(u, v)$.

Suppose the three following conditions are all satisfied:

(a) For each positive integer $N$, there exists a continuous function $C_{N}(r)$ on $R^{1}$ with $C_{N}(r)>0$ for $r>0, \lim C_{N}(r)=+\infty$ as $r \rightarrow+\infty$ such that

$$
\operatorname{Re}\left(G_{u} v-G_{u} w, v-w\right) \geqq C_{N}\left(\|v-w\|_{X}\right) \cdot\left\|_{v}-w\right\|_{X}
$$

for all $u$ in $Y$ with $\|u\|_{Y} \leqq N$ and all $v$ and w in $X$.

(b) There exists a continuous real-valued function $c(r)$ on $R^{1}$ with $\lim c(r)=+\infty$ as $r \rightarrow+\infty$ such that for all $k \geqq 1$,

$$
\operatorname{Re}\left(G_{u}(k u), u\right) \geqq c\left(\|u\|_{X}\right)\|u\|_{X}
$$

for all $u$ in $X$.

(c) For each $u$ in $Y, G_{u}$ is continuous from the strong topology of $X$ 
to the weak topology of $X^{*}$. For each fixed $v$ in $X$, the map $u \rightarrow G_{u} v$ is a strongly continuous mapping from $Y$ to $X^{*}$.

Then for every w in $X^{*}$, there exists $u$ in $X$ such that $G_{u}(u)=w$.

2. Existence theorems for nonlinear equations involving monotone operators were proved by the writer in [1] for separable reflexive Banach spaces and slightly later but independently by G. J. Minty [5] for reflexive Banach spaces without a separability assumption. These results use very little of the Banach space structure and have the following generalization to locally convex spaces.

Let $E_{1}$ and $E_{2}$ be two locally convex linear Hausdorff spaces over the reals. Let $\left(E_{1}, E_{2}\right)$ be a dual system, i.e., for $u$ in $E_{1}$ and $v$ in $E_{2}$ we have a bilinear pairing $(u, v)$ defined such that: $(u, v)$ is continuous in each variable, with the other held fixed and if $v$ in $E_{2}$ annihilates all $v$ in $E_{1}$, then $v=0$. If $T$ is a map from $E_{1}$ to $E_{2}, T$ is said to be monotone if $(u-v, T u-T v) \geqq 0$ for all $u$ and $v$ in $E_{1} . T$ is said to be finitely continuous if it is continuous from every finite-dimensional subspace of $E_{1}$ to $E_{2}$.

Let $S$ be a subset of $E_{1} . K(S)$ denotes the closed convex hull of $S$ in $E_{1}$. If $u \in K(S)-S, S$ is said to envelop $u$ if for every finitedimensional flat $F$ containing $u$, the boundary of $K(S) \cap F$ is contained in $S \cap F$.

If $C$ is a map of $E_{1}$ into $E_{2}, C$ is said to be completely continuous (with respect to $\left(E_{1}, E_{2}\right)$ ) if $C$ is continuous and if the map $u \rightarrow(u, C u)$ is a continuous map from each compact subset of $E_{1}$ to $R^{1}$.

Theorem 3. Let $\left(E_{1}, E_{2}\right)$ be a dual system, $T$ a map of $E_{1}$ into $E_{2}$ such that $T=T_{0}+C$ where $T_{0}$ is finitely continuous and monotone, $C$ completely continuous. Let $S \subset E_{1}$ be such that $K(S)$ is compact, $u_{0} a$ point of $K(S)-S$ such that $S$ envelops $u_{0}$. Suppose that for given w in $E_{2}$, we have

$$
\left(u-u_{0}, T u-w\right) \geqq 0
$$

for all $u$ in $S$.

Then there exists $u_{1}$ in $K(S)$ such that $T u_{1}=w$.

Specializing Theorem 3, we get the two following theorems.

Theorem 4. Let $X$ be a real Banach space, $X^{*}$ its adjoint space, $T$ a map of $X^{*}$ into $X$ such that $T=T_{0}+C$, where $T_{0}$ is monotone, $T_{0}$ is continuous from finite-dimensional subspaces of $X^{*}$ to the weak topology of $X, C$ is continuous on bounded subsets of $X^{*}$ from the weak* topology of $X^{*}$ to the strong topology of $X$. Let $S$ be a bounded subset of $X^{*}$ which envelops $u_{0}$ in $X^{*}$, w $\in X$ such that 


$$
\left(u-u_{0}, T u-w\right) \geqq 0
$$

for all $u$ in $S$. Then if $K(S)$ is the w* closed convex hull of $S$, there exists $u_{1}$ in $K(S)$ such that $T u_{1}=w$.

Theorem 5. Let $X$ be a reflexive B-space, $Y$ a Banach space, $T$ a map of $X$ into $Y^{*}$ such that $T=T_{0}+C$ where $T_{0}$ is continuous from finitedimensional subspaces of $X$ to the weak* topology of $Y^{*}, C$ continuous on bounded sets of $X$ from the weak topology of $X$ to the strong topology of $Y^{*}$.

Suppose that there exists a bounded linear operator $L$ from $X$ to $Y$ with dense range in $Y$ such that

$$
\left(T_{0} u-T_{0} v, L_{0} u-L_{0} v\right) \geqq 0
$$

for all $u$ and $v$ in $X$. Let $S$ be a bounded subset of $X$ which envelops a point $u_{0}$ of $K(S)$, the closed convex hull of $S$, and such that for a given w in $Y^{*}$,

$$
\left(T u-w, L u-L u_{0}\right) \geqq 0
$$

for all $u$ in $S$.

Then there exists $u_{1}$ in $K(S)$ such that $T u_{1}=w$.

\section{BIBLIOGRAPHY}

1. F. E. Browder, Nonlinear elliptic boundary value problems, Bull. Amer. Math. Soc. 69 (1963), 862-874.

2. - Nonlinear parabolic boundary value problems of arbitrary order, Bull. Amer. Math. Soc. 69 (1963), 858-861.

, Strongly nonlinear parabolic boundary value problems, Amer. J. Math. (to appear).

4. —_, Nonlinear elliptic boundary value problems. II, Trans. Amer. Math. Soc. (to appear).

5. G. J. Minty, On a "monotonicity" method for the solution of nonlinear equations in Banach space, Proc. Nat. Acad. Sci. (to appear).

6. M. I. Visik, Solution of a system of quasilinear equations in divergence form under periodic boundary conditions, Dokl. Akad. Nauk SSSR 137 (1961), 502-505. (Russian)

7. - Boundary value problems for quasilinear strongly elliptic systems in divergence form, Dokl. Akad. Nauk SSSR 138 (1961), 518-521. (Russian)

8. - Simultaneous quasilinear equations with lower order terms, Dokl. Akad. Nauk SSSR 144 (1962), 13-16. (Russian)

9. - Quasilinear strongly elliptic systems of differential equations having divergence form, Trudy Moskov Mat. Obšč. 12 (1963), 125-184.

Institute for Advanced Study AND University of Chicago 